\title{
Cartographies participatives : diversité des situations, diversité des pratiques
}

Participatory mapping: the diversity of situations and practices

Pascale de Robert, Stéphanie Duvail et Élisabeth Habert

\section{(2) OpenEdition}

\section{Journals}

\section{Édition électronique}

URL : http://journals.openedition.org/ethnoecologie/3048

DOI : 10.4000/ethnoecologie.3048

ISSN : 2267-2419

Éditeur

Laboratoire Eco-anthropologie et Ethnobiologie

\section{Référence électronique}

Pascale de Robert, Stéphanie Duvail et Élisabeth Habert, «Cartographies participatives : diversité des situations, diversité des pratiques », Revue d'ethnoécologie [En ligne], 11 | 2017, mis en ligne le 03 juillet 2017, consulté le 24 septembre 2020. URL : http://journals.openedition.org/ethnoecologie/3048 ;

DOI : https://doi.org/10.4000/ethnoecologie.3048

Ce document a été généré automatiquement le 24 septembre 2020.

\section{c) (7) $(-)$}

Revue d'ethnoécologie est mis à disposition selon les termes de la licence Creative Commons Attribution - Pas d'Utilisation Commerciale - Pas de Modification 4.0 International. 


\title{
Cartographies participatives : diversité des situations, diversité des pratiques
}

\author{
Participatory mapping: the diversity of situations and practices
}

Pascale de Robert, Stéphanie Duvail et Élisabeth Habert

1 Dans un premier dossier «Cartographie participative " publié dans le numéro 9, nous avons insisté sur les enjeux forts de la "mise en carte" aux Suds (Robert \& Duvail 2016). La cartographie de territoires habités par des populations restées en marge des centres de décision, ou porteurs de ressources et de projets disputés, est un exercice qui a des implications politiques en ce qu'il délimite, donne à voir ou officialise une version $d u$ territoire. Les chercheurs ne sauraient donc s'engager dans une cartographie dite " participative " sans analyser en amont le contexte de tels travaux à l'échelle locale, nationale et internationaleet sans en saisir les tenants et aboutissants pour chacune des parties prenantes. Dans le premier dossier nous avions ainsi souligné le rôle de la carte comme révélateur (ou catalyseur) de conflits ouverts ou latents en même temps que les limitations bien souvent observées des outils cartographiques actuels. Les exemples développés insistaient également sur l'importance des relations de confiance préexistantes à l'exercice de la cartographie entre population locale et chercheurs.

Dans ce deuxième dossier, nous présentons de nouvelles expériences de cartographie menées par des groupes d'habitants de différents pays, sur plusieurs continents (au Sénégal, en Guyane française, au Brésil, aux Marquises, au Kenya et au Timor oriental) en collaboration avec des chercheurs venus de diverses disciplines (géographie, cartographie, archéologie, ethnologie, linguistique, histoire). Ces travaux sont illustratifs de la variété des situations qui président à l'organisation d'un exercice de cartographie (participative) et, en conséquence, reflètent la diversité des pratiques mises en œuvre pour produire ensemble une représentation cartographique du territoire. La multiplicité des approches et des méthodes, la souplesse et la créativité des moyens mis en œuvre pour «ajuster » les outils aux situations rencontrées sont 
nécessaires. Pour autant, elles ne garantissent pas toujours des résultats satisfaisants dans les contextes fonciers, économiques et politiques parfois difficiles de certains terrains : la carte peut mener tout autant à une impasse voire à un conflit, qu'à un consensus fédérateur. Elle n'en reste pas moins un outil de grand intérêt pour réfléchir au territoire et à ses transformations dans le temps en intégrant les perspectives de plusieurs disciplines, plusieurs langues, et plusieurs projets de gestion.

Ces différentes expériences, qui donnent un éventail significatif de la diversité des situations et des pratiques de cartographie participative aux Suds, ont été discutées au sein de l'atelier "Cartographies participatives" organisé par l'UMR «Patrimoines Locaux et Gouvernance » durant l'année 2016-2017.

\section{BIBLIOGRAPHIE}

Robert P. de \& Duvail S. 2016 - « Mettre en carte » le territoire Revue d'ethnoécologie [En ligne], 9| 2016, mis en ligne le 01 juillet 2016, consulté le 27 mars 2017. URL : http://

ethnoecologie.revues.org/2739; DOI : 10.4000/ethnoecologie.2739

\section{AUTEURS}

\section{STÉPHANIE DUVAIL}

Chargée de Recherche IRD - UMR 208 « Patrimoines locaux et gouvernance » (PALOC)- Muséum national d'Histoire naturelle Paris, France

stephanie.duvail@ird.fr

\section{ÉLISABETH HABERT}

Ingénieur cartographe - UMR 208 IRD-MNHN "Patrimoines locaux et gouvernance" (PALOC) MNHN, Département HNS - 57 rue Cuvier, CP 51, 75231 Paris Cedex 05, France

elisabeth.habert@ird.fr 